\section{JTI}

JOURNAL OF

TRAUMA AND INJURY

\title{
Clinical Outcomes of Diffuse Axo- nal Injury According to Radiological Grade
}

Hak-Jae Lee, M.D., Hyun-Woo Sun, M.D., Jae-Seok Lee, M.D., Nak-Joon Choi, M.D., Yoon-Joong Jung, Suk-Kyung Hong, M.D., Ph.D.

Division of Acute Care Surgery, Department of Surgery, Asan Medical Center, University of Ulsan College of Medicine, Seoul, Korea

Received: March 23, 2018

Revised: June 18, 2018

Accepted: July 6, 2018

\section{Correspondence to}

Suk-Kyun Hong, M.D., Ph.D.

Division of Acute Care Surgey, Department of Surgery, Asan Medical Center, University of Ulsan College of Medicine, 88 Olympic-ro 43-gil, Songpa-gu, Seou 05505, Korea

Tel: $+82-2-3010-6700$

Fax: +82-2-3010-6701

E-mail:skhong94@amc.seoul.kr
Purpose: Patients with diffuse axonal injury experience various disabilities and have a high cost of treatment. Recent researches have revealed the underlying mechanism and pathogenesis of diffuse axonal injury. This study aimed to investigate the correlation between the radiological grading of diffuse axonal injury and the clinical outcomes of patients.

Methods: From January 2011 to December 2016, among 294 patients with traumatic brain injury, 44 patients underwent magnetic resonance imaging (MRI). A total of 18 patients were enrolled in this study except for other cerebral injuries, such as cerebral hemorrhage or hypoxic brain damage. Demographic data, clinical data, and radiological findings were retrospectively reviewed. The grading of diffuse axonal injury was analyzed based on patient's MRI findings.

Results: For the most severe diffuse axonal injury patients, prolonged intensive care unit (ICU) stay ( $p=0.035)$, hospital stay $(p=0.012$ ), and prolonged mechanical ventilation $(p=0.030)$ were observed. However, there was no significant difference in healthcare-associated infection rates between MRI grading ( $p=0.123$ ). Massive transfusion, initial hemoglobin and lactate levels, and MRI gradings were found to be highly significant in predicting the duration of unconsciousness.

Conclusions: This study showed that patients with high grade diffuse axonal injury have prolonged ICU stays and significantly longer hospital stays. Deteriorated mental patients with high energy injuries should be evaluated to identify diffuse axonal injuries by using an appropriate imaging tool, such as MRI. It will be important to predict the duration of consciousness recovery using MRI scans.

Keywords: Brain injury, Traumatic; Diffuse axonal injury; Magnetic resonance imaging; Prognosis 


\section{JTI}

\section{INTRODUCTION}

Diffuse axonal injury (DAI) causes severe disability and mortality following closed head trauma, especially after traffic accidents. DAI is caused by widespread damage to axons following a closed head injury. It was first defined by Strich [1] and the structure and course of DAI were reviewed by Adams et al. [2]. Many studies have been carried out on the pathogenesis and biomechanics of DAI $[3,4]$. Axonal damage was produced by mechanical forces that sheared the fibers at the moment of impact [5]. Notably, diffuse damage can be caused by the angular or rotational acceleration-deceleration forces that occur in traffic accidents [6,7]. Many DAI patients experience a prolonged coma and various sequelae, such as hospital-acquired infection. Therefore, it is important to use magnetic resonance imaging (MRI) for patients who do not recover consciousness in absence of focal lesions in the brain. Some studies suggest that the degree of injury based on MRI findings is related to the time taken to recover consciousness $[8,9]$. As the duration of coma is often longer in DAI patients, more complications related DAI will arise. This study aimed to investigate the correlation between the severity of DAI based on MRI grading and clinical outcomes, such as time to recover consciousness, the duration of intensive care unit (ICU), and hospital stays, and complication rates.

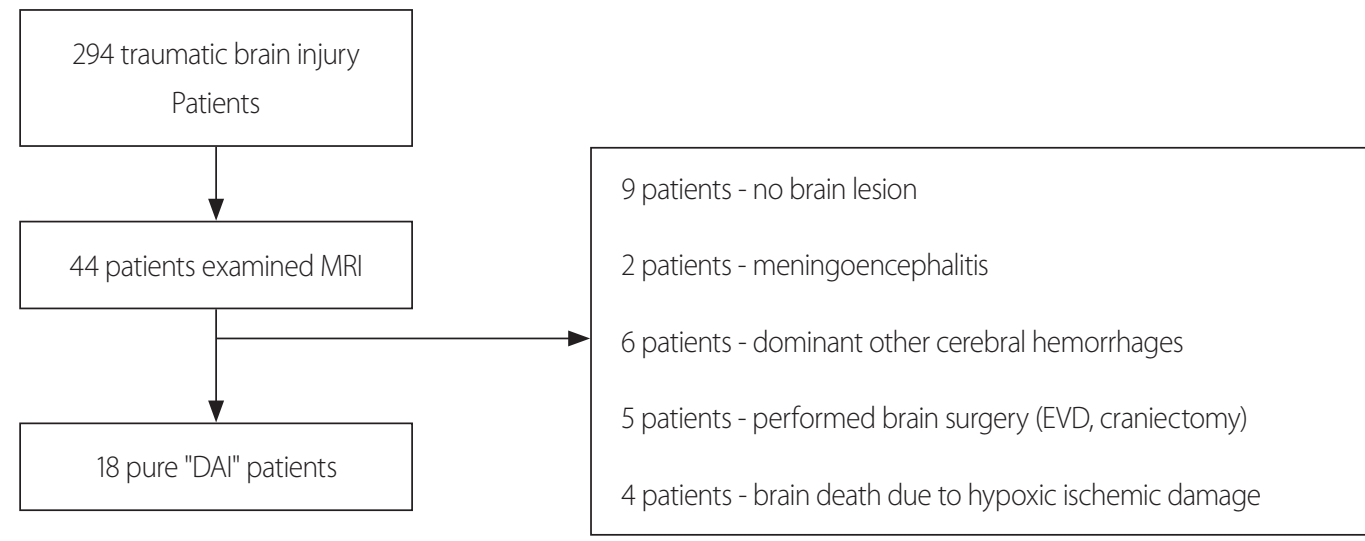

Fig. 1. Participating patients in this study. MRI: magnetic resonance imaging, DAl: diffuse axonal injury, EVD: external ventricular drain.

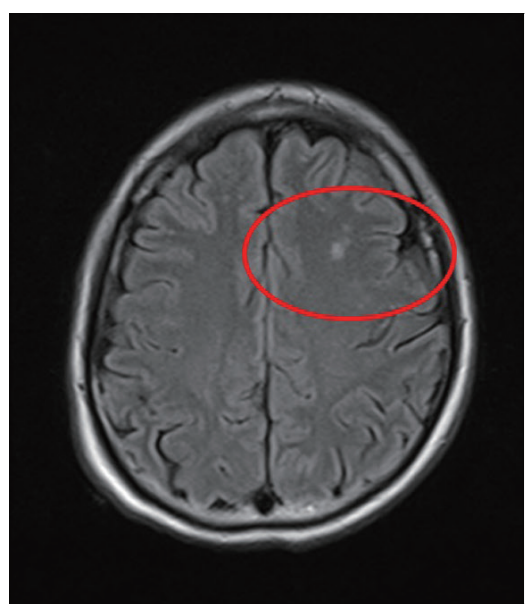

Grade I

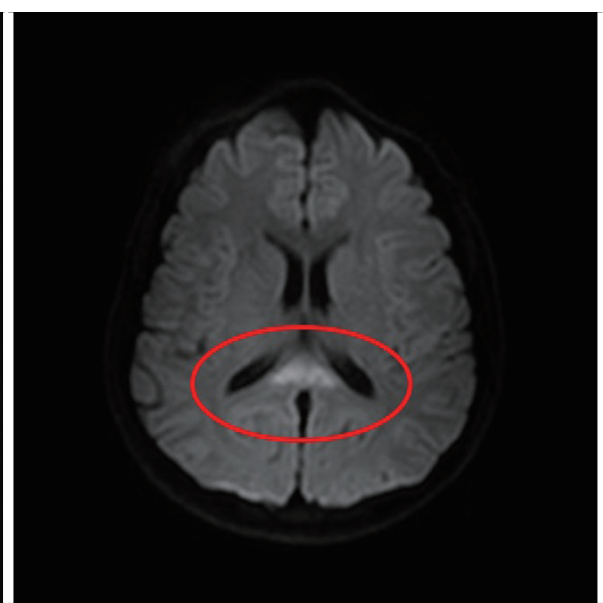

Grade II

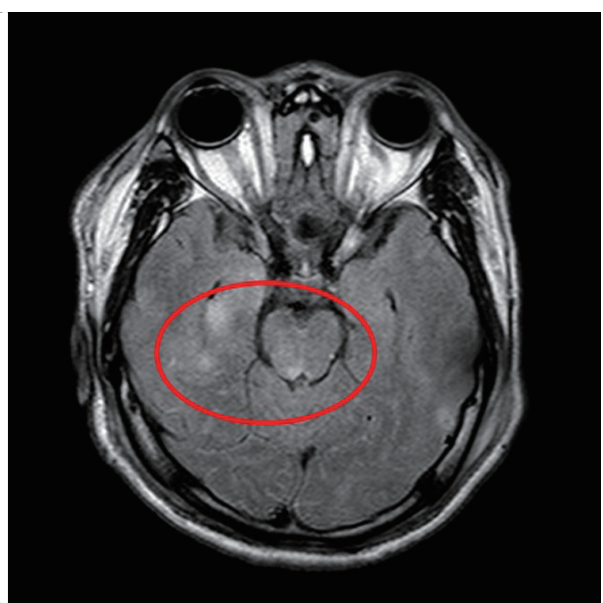

Grade III

Fig. 2. MRI show punctuate microhemorrhage on the white matter, corpus callosum and mid-brain, respectively (Grade l: the bleeding is limited within cerebral cortex. Grade II: the bleeding is limited within corpus callosum. Grade III: the bleeding is extended to the midbrain. Each circle represents microhemorrhage). MRI: magnetic resonance imaging. 


\section{METHODS}

A total of 294 patients were diagnosed with traumatic brain injury from January 2011 to December 2016.

Table 1. Demographic and clinical characteristics of patients with diffuse axonal injury

\begin{tabular}{|lc|}
\hline Characteristics & $\mathbf{N}(\%)$ \\
\hline Age (years) & $2(11.1 \%)$ \\
$\leq 20$ & $8(44.4 \%)$ \\
$21-40$ & $5(27.8 \%)$ \\
$41-60$ & $3(16.7 \%)$ \\
$\geq 61$ & $16(88.9 \%)$ \\
Gender (male, \%) & \\
Mechanism & $4(22.2 \%)$ \\
Pedestrian TA & $3(16.7 \%)$ \\
In car TA & $1(5.6 \%)$ \\
Bicycle TA & $4(22.2 \%)$ \\
Motorcycle TA & $6(33.3 \%)$ \\
Fall down & \\
Grade & $7(38.9 \%)$ \\
I & $7(38.9 \%)$ \\
II & $4(22.2 \%)$ \\
III & $5(27.7 \%)$ \\
Hypotension (below 90 mmHg sBP) & $2(11.1 \%)$ \\
Massive transfusion (within 24 hours, above 10 packs) & $10.72 \pm 3.44$ \\
GCS & $27.56 \pm 11.28$ \\
ISS & $12.99 \pm 2.30$ \\
Initial hemoglobin (g/dL) & $3.49 \pm 2.47$ \\
Initial lactate (mmol/L) & \\
\hline
\end{tabular}

TA: traffic accident, sBP: systolic blood pressure, GCS: Glasgow coma scale, ISS: injury severity score.
Among these patients, 44 patients underwent MRI because of their clinical presentation. We excluded those patients with other diagnoses, such as hypoxic brain damage or other dominant cerebral hemorrhage. Additionally, patients who had undergone a cranial operation during the admission period were excluded. Ultimately, 18 patients with "pure DAI" were enrolled (Fig. 1). All medical records and radiographic findings were reviewed retrospectively.

Based on MRI imaging, patients with pure DAI were divided into three grades according to the Adams anatomical grading system [2,9]. The main imaging features of DAI is punctuated microhemorrhage. As shown in the Fig. 2, grade I is the case where the bleeding is limited within cerebral cortex. Grade II is a case of bleeding to corpus callosum. Grade III is the case of bleeding extended to the midbrain (Fig. 2). Demographic data and clinical information were collected from electrical medical records. One radiologist reviewed all the radiologic findings. The MRI sequence used for the grading was mainly diffusion-weighted sequence ( 9 cases), T2-fluid attenuated inversion recovery (FLAIR) (7 cases) and T2 gradient echo imaging ( 2 cases). The initial lab finding (hemoglobin and lactate) and Glasgow coma scale (GCS) were measured at the time of arrival at the emergency room. Notably, the time required to recover consciousness was defined as the period until simple orders could be obeyed while the patient's eyes were open.

We used the nonparametric Kruskal-Wallis test for continuous variables, because of the skewed data distribution. Bonferroni correction was used for post-hoc

Table 2. Clinical outcomes of diffuse axonal injury by MRI grading

\begin{tabular}{|c|c|c|c|c|}
\hline & Grade I $(n=7)$ & Grade II $(n=7)$ & Grade III $(n=4)$ & $p$-value \\
\hline Age (years) & $49.3 \pm 18.3$ & $38.7 \pm 21.4$ & $29.5 \pm 12.0$ & 0.092 \\
\hline GCS & $11.7 \pm 2.3$ & $11.3 \pm 3.6$ & $8.0 \pm 4.2$ & 0.112 \\
\hline Initial hemoglobin (g/dL) & $13.6 \pm 1.1$ & $13.6 \pm 1.0$ & $10.8 \pm 4.2$ & 0.077 \\
\hline Initial lactate (mmol/L) & $3.1 \pm 1.3$ & $3.1 \pm 1.7$ & $4.9 \pm 4.7$ & 0.303 \\
\hline Time to recover consciousness (days) & $5.0(2.0,9.5)$ & $14.0(9.5,35.0)$ & $28.0(20.5,43.5)$ & 0.019 \\
\hline ICU stay (days) & $5.0(1.5,11.5)$ & $12.0(9.5,34.0)$ & $24.5(20.0,40.5)$ & 0.035 \\
\hline Length of hospital stay (days) & $15.0(6.0,22.5)$ & $57.0(32.0,78.5)$ & $52.0(33.5,88.5)$ & 0.012 \\
\hline Duration of mechanical entilation (days) & $4.0(0.0,7.0)$ & $11.0(6.0,25.5)$ & $23.5(19.0,33.5)$ & 0.030 \\
\hline
\end{tabular}

MRI: magnetic resonance imaging, GCS: Glasgow coma scale, ICU: intensive care unit. 
analysis. Additionally, we used the chi-square and Fisher exact tests to compare proportions of application of tracheostomy and complication rates. A linear backwards regression analysis was performed to predict the duration of coma based on clinical variables that were considered to be prognostic factors. Statistical analyses were conducted with $\mathrm{R}$ program ( $\mathrm{R}$ program version 3.3.2, R Foundation for Statistical Computing, Vienna, Austria).

\section{RESULTS}

Among 18 patients with DAI, 16 (88.9\%) were men, and two $(11.1 \%)$ were women. The ages at the time of injury ranged from 16 to 76 years with a mean age of 40.78 years. There were 12 patients who experienced traffic accidents, and the others were injured by falling. None of the patients died. According to MRI grading system, seven patients (38.9\%) had grade I injuries, seven patients (38.9\%) had grade II injuries, and four patients (22.2\%) had grade III injuries. At the time of admission, a total of 5 patients had hypotension, and two patients received massive red blood cells (RBC) transfusion (more than 10 packs). The mean GCS and injury severity score (ISS) were 10.72 \pm 3.44 and 27.56 \pm 11.28 , respectively. Additionally, mean lactate $(\mathrm{mmol} / \mathrm{L})$ and hemoglobin $(\mathrm{g} / \mathrm{dL})$ levels were $3.49 \pm 2.47$ and $12.99 \pm 2.30$, respectively (Table 1 ).

The mean time to recover consciousness was 20.33 days.
The median time to regain awake status was significantly different between grade I, II, and III patients $(p=0.019)$. Those patients within the grade I group required 5 days to recover consciousness, while those patients classified as grades II and III required 2 and 4 weeks, respectively. As the grading of MRI increased, the duration of ICU stay $(p=0.035)$ and hospitalization $(p=0.012)$ also increased. Finally, the duration of mechanical ventilation increased according to patient's MRI grading ( $p=0.030$ ) (Table 2).

The time required to recover consciousness (grade I vs. grade II; $p=0.018$, grade II vs. III; $p=0.032$ ) and the ICU stay period (grade I vs. grade II; $p=0.029$, grade II vs. III; $p=0.036$ ) differed for each grade, (grade I vs. grade II; $p=0.029$, grade II vs. III; $p=0.036$ ) but the length of hospital stay significantly differed between grades I and II $(p=0.054)$. There was no significant difference between grades II and III (Fig. 3).

We also examined the application of tracheostomy and healthcare-associated infection rates according to MRI grading. There were significant differences for the application of tracheostomy according to MRI grading ( $p=0.024$ ). Additionally, among patients with DAI, nine patients had healthcare-associated infections, including central line-associated blood stream infection (5 cases), and ventilator-associated pneumonia (4 cases). There were no significant differences in complication rates according to grade $(p=0.123)$ (Table 3$)$.

Among patients with traumatic brain injury, the prog-
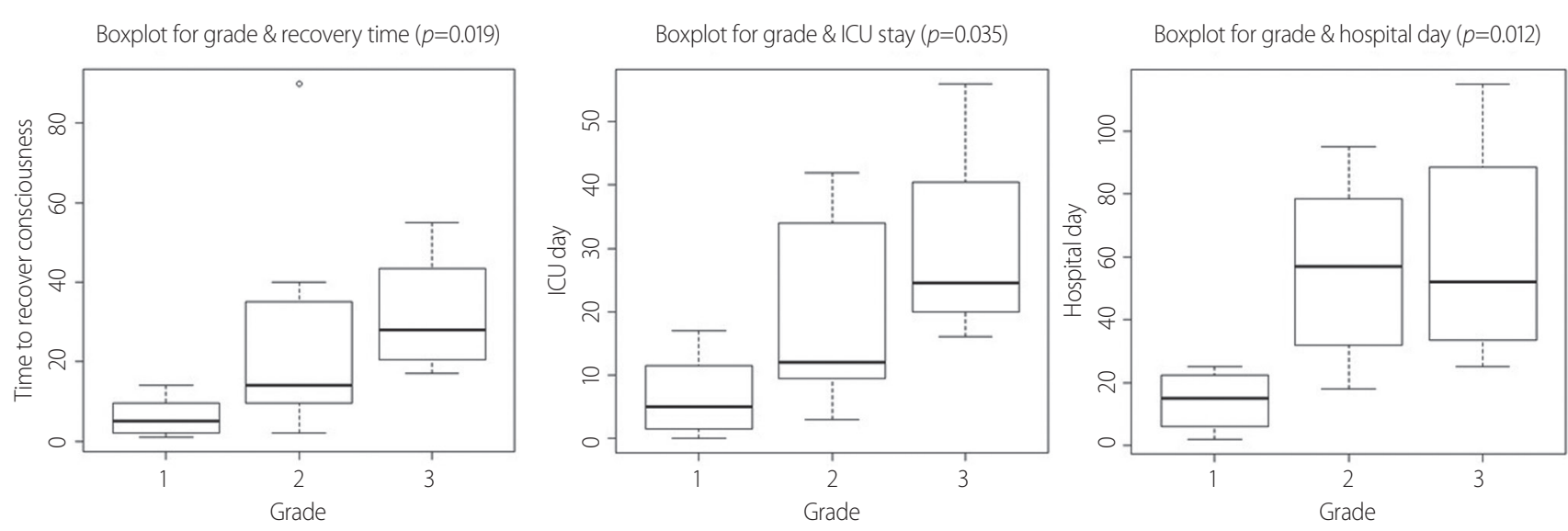

Fig. 3. Associations of the recovery time, ICU stay, and hospital LOS with MRI grade. The following boxplots show that there is a difference in time to recover consciousness and ICU day according to each grade. But, there is no difference between grade II and III at length of hospital day. ICU: intensive care unit, LOS: length of stay, MRI: magnetic resonance imaging. 
nostic factors that affect the time taken to recover consciousness were initial hemoglobin, initial lactate level, and MRI grade II $(p=0.041)$. The characteristics included in this analysis were age, GCS, anisocoria, hypotension, massive transfusion (or not), initial hemoglobin and lactate levels, and MRI grade (Table 4).

\section{DISCUSSION}

Since the initial report was published by Strich [1], many studies have examined the pathogenesis and clinical outcomes of DAI. The development of radiological modalities has led to major changes in the diagnosis of DAI. Because affected patients require long recovery periods and rehabilitation times and high treatment costs, trauma physicians need to make accurate predictions about the clinical course $[3,10]$.

Park et al. [9] reported a positive correlation between MRI grade and the duration of unconsciousness. They found that grade I patients had a 1 week recovery time, whereas the recovery of grade II and III patients took 2 weeks and 2 months, respectively. Our study showed similar results (grade I-5 days; grade II-2 weeks; grade III-
1 month). However, in other studies $[9,11,12]$, there were more patients in the grade II or III group, whereas more patients in our current study were classified as grade I or II. This finding suggests that differences in the sequencing of MRI methods may have led to these results. Recent studies [12-14] have shown a higher sensitivity as MRI sequences have developed. Mac Donald et al. [15] suggested that diffusion tensor imaging (DTI) may be a more sensitive method to evaluate DAI lesions than conventional MRI, and that it may have a high negative predictive value. Also, Ashwal et al. [16] reported that the detection rates of hemorrhagic lesions in susceptibility weighted MR imaging (SWI) were six times greater than those from conventional MRI. However, MRI can be difficult to perform on DAI patients because they have multiple accompanying injuries and unstable vital signs. Thus, whether to perform MRI should be considered carefully according to the condition of the patient.

Many previous studies have examined the prognostic factors associated with DAI. Zheng et al. [8] reported that the number of cerebral lesions and age, as well as ADC (apparent diffusion coefficient) scores from MRI scans were highly significant in predicting coma duration. In our current analysis, we found that massive transfusion,

Table 3. Application of tracheostomy and hospital-acquired infection rates according to MRI grade

\begin{tabular}{|lcccc|}
\hline & Grade I & Grade II & Grade III & p-value \\
\hline Application of tracheostomy & $14.3 \%(1 / 7)$ & $57.1 \%(4 / 7)$ & $100 \%(4 / 4)$ & 0.024 \\
Hospital-acquired infection & $28.6 \%(2 / 7)$ & $57.1 \%(4 / 7)$ & $75.0 \%(3 / 4)$ & 0.123 \\
& VAP-2 cases & VAP-3 cases & CLABSI-3 cases \\
& & CLABSI-1 case & & \\
\hline
\end{tabular}

MRI: magnetic resonance imaging, VAP: ventilator-associated pneumonia, CLABSI: central line associated blood stream infection.

Table 4. Prognostic factors associated with recovery of consciousness (multivariate regression analysis, backward selection)

\begin{tabular}{|lcc|}
\hline & Regression coefficient $(\mathbf{9 5 \%}$ confidence interval) & $p$-value \\
\hline Massive transfusion & $-66.683(-113.023,-20.343)$ & 0.008 \\
Initial hemoglobin $(\mathrm{g} / \mathrm{dL})$ & $-11.015(-18.597,-3.432)$ & 0.008 \\
Initial lactate $(\mathrm{mmol} / \mathrm{L})$ & $9.404(2.136,16.671)$ & 0.015 \\
Grade II (reference: grade I) & $31.578(11.145,52.011)$ & 0.005 \\
Grade III (reference: grade I) & $-5.651(-39.508,28.205)$ & 0.722 \\
\hline
\end{tabular}

Dependent variables - age, GCS, anisocoria, hypotension, massive transfusion (more than 10 packs), hemoglobin, lactate, and MRI grade. $p$-value=0.021, adjusted $R_{2}=0.474$.

GCS: Glasgow coma scale, MRI: magnetic resonance imaging. 
higher initial lactate and lower hemoglobin levels, and higher MRI grades were associated with delayed recovery of consciousness. Therefore, we could expect that recovery would be delayed when a patient is admitted with a severe condition.

In our study, all patients recovered consciousness. In particular, patients with grade III were more severe cases at the time of injury. However, the average age of the patients with grade III were 29.5 years, and we suggested that they could have recovered consciousness.

Our study also showed that the application of tracheostomy has increased along with increasing grades. However, the reasons for this finding should be carefully considered because there may be differences depending upon variations in treatments between hospitals. In patients with DAI, a higher MRI grade will result in a longer duration of treatment. Therefore, more active treatment, such as early tracheostomy application, should be administered.

We analyzed the factors affecting recovery of consciousness and analyzed the incidence of healthcare-associated infection due to delayed recovery of consciousness. So, it is necessary to measure the degree of DAI through MRI in patients with traumatic brain injury (TBI) who have severe injury and massive transplantation.

There were several limitations to this study. First, we did not examine MRI findings according to the protocol that was prescribed. As a consequence, there was a difference in the timing and sequencing of MRI examinations. Although the examination is performed according to the condition of the patient, there may be differences in the results of these different studies. Second, the number of patients in this study was small. More patients were clinically suspected, but we confined our analysis to those patients who underwent MRI scans in order to minimize bias. Indeed, there were more patients suspected of DAI by clinical findings or computed tomography (CT) imaging. However, we had to limit the enrolled patients because they could bias the diagnostic results. Most DAI patients usually required ventilator and ICU care. Therefore, although the sample size is small, the following study may be helpful in establishing a prognosis or treatment plan by examining MRI in patients suspected of having DAI.

\section{CONCLUSION}

DAI is major mechanisms involved in the traumatic brain injury. In affected patients, long-term hospitalization is necessary until consciousness is restored, and this period is associated with the many problems of ICU care. Therefore, it will be important to predict the duration of consciousness recovery using MRI scans. MRI examinations in DAI patients can help to predict the prognosis of a patient and to establish a treatment direction under a systematic protocol

\section{ACKNOWLEDGEMENTS}

This study was presented at the Annual Congress of the Korean Society of Acute Care Surgery on April 7, 2017 in Seoul, Korea.

\section{REFERENCES}

1. Strich SJ. Diffuse degeneration of the cerebral white matter in severe dementia following head injury. J Neurol Neurosurg Psychiatry 1956;19:163-85.

2. Adams JH, Doyle D, Ford I, Gennarelli TA, Graham DI, McLellan DR. Diffuse axonal injury in head injury: definition, diagnosis and grading. Histopathology 1989;15:49-59.

3. Meythaler JM, Peduzzi JD, Eleftheriou E, Novack TA. Current concepts: diffuse axonal injury-associated traumatic brain injury. Arch Phys Med Rehabil 2001;82:1461-71.

4. Smith DH, Hicks R, Povlishock JT. Therapy development for diffuse axonal injury. J Neurotrauma 2013;30:307-23.

5. Blumbergs PC, Jones NR, North JB. Diffuse axonal injury in head trauma. J Neurol Neurosurg Psychiatry 1989;52:838-41.

6. Gennarelli TA, Thibault LE, Adams JH, Graham DI, Thompson CJ, Marcincin RP. Diffuse axonal injury and traumatic coma in the primate. Ann Neurol 1982;12:564-74.

7. Adams JH. Diffuse axonal injury in non-missile head injury. Injury 1982;13:444-5.

8. Zheng WB, Liu GR, Li LP, Wu RH. Prediction of recovery from a post-traumatic coma state by diffusion-weighted imaging (DWI) in patients with diffuse axonal injury. Neuroradiology 2007;49:271-9. 
9. Park SJ, Hur JW, Kwon KY, Rhee JJ, Lee JW, Lee HK. Time to recover consciousness in patients with diffuse axonal injury : assessment with reference to magnetic resonance grading. J Korean Neurosurg Soc 2009;46:205-9.

10. Kim JH, Sung SK, Cho WH, Choi CH. Disability estimation in the diffuse axonal injury. J Korean Neurotraumatol Soc 2007;3:13-8.

11. Chelly H, Chaari A, Daoud E, Dammak H, Medhioub F, Mnif J, et al. Diffuse axonal injury in patients with head injuries: an epidemiologic and prognosis study of 124 cases. J Trauma 2011;71:838-46.

12. Abu Hamdeh S, Marklund N, Lannsjö M, Howells T, Raininko R, Wikström J, et al. Extended anatomical grading in diffuse axonal injury using MRI: hemorrhagic lesions in the substantia nigra and mesencephalic tegmentum indicate poor long-term outcome. J Neurotrauma 2017;34:341-52.

13. Jung NK, Jin SC, Choi WI. Prognostic value of computed to- mography and gradient-echo magnetic resonance imaging in diffuse axonal injury. J Trauma Inj 2012;25:122-31.

14. Moen KG, Brezova V, Skandsen T, Håberg AK, Folvik M, Vik A. Traumatic axonal injury: the prognostic value of lesion load in corpus callosum, brain stem, and thalamus in different magnetic resonance imaging sequences. J Neurotrauma 2014;31:148696.

15. Mac Donald CL, Dikranian K, Song SK, Bayly PV, Holtzman DM, Brody DL. Detection of traumatic axonal injury with diffusion tensor imaging in a mouse model of traumatic brain injury. Exp Neurol 2007;205:116-31.

16. Ashwal S, Babikian T, Gardner-Nichols J, Freier MC, Tong KA, Holshouser BA. Susceptibility-weighted imaging and proton magnetic resonance spectroscopy in assessment of outcome after pediatric traumatic brain injury. Arch Phys Med Rehabil 2006;87(12 Suppl 2):S50-8. 